\title{
A Non-Interruptive Link-Variation Monitoring Circuit for Wireless Sensor Applications
}

\author{
Stephen T. Kim, Student Member, IEEE, Jaehyouk Choi, Kwanyeob Chae, Sungho Beck, Seong-Hyok Kim,
} Franklin Bien, Member, IEEE, Chang-Ho Lee, Senior Member, IEEE, Kyutae Lim, Member, IEEE, Joy Laskar, Fellow, IEEE, and Manos M. Tentzeris, Fellow, IEEE

\begin{abstract}
As wireless sensor devices are usually deployed where the operating environment changes unpredictably, the ability to evaluate the variation in the wireless link is required for reliable and efficient operations. In this paper, a novel link-variation-to-digital-converter (LDC) that provides real-time assessments on the link variations is proposed. Due to its unique structure, the proposed LDC can adjust the resolution of the output data and does not interrupt the original system functionality. A wireless power receiver prototype with a proposed circuit is implemented in CMOS $0.18 \mu \mathrm{m}$ with an active area of $500 \mu \mathrm{m}$-by-20 $\mu \mathrm{m}$. Measurements of the prototype show that link-variations due to mismatches in the resonant matching condition as well as physical misalignments can be successfully evaluated with the proposed LDC.
\end{abstract}

Index Terms-Adaptive matching, link variation, resonant matching, wireless power transmission.

\section{INTRODUCTION}

A DVANCED CMOS technology has enabled integration of multiple functions in a single-chip device that can still maintain reasonably low-power as well as low-cost. With these remarkable accomplishments, the concept of ubiquitous sensing and wireless communication with a micro-scale sensor device was introduced to military, commercial, as well as medical applications [1], [2]. Depending on applications, the core functional blocks of sensor devices are designed to observe different kinds of physical phenomenon such as pressure, brightness, and temperature, and it is highly desirable to maximize the system efficiency of the core blocks. However, the efficiency upper bounds cannot promise consistent performance because a wireless sensor device must undergo variations in the operating environments as the device is deployed. For example, changes in either the physical distance or orientation would attenuate or intensify incoming and out-going signals. Consequently, unless these inevitable yet unpredictable link variations are properly

\footnotetext{
Manuscript received February 20, 2011; revised August 28, 2011; accepted September 27, 2011. Date of publication November 07, 2011; date of current version December 07, 2011. This work was supported in part by the Interconnect Focus Center, one of the Semiconductor Research Corporation Focus Centers.

S. T. Kim, K. Chae, S.-H. Kim, K. Lim, J. Laskar, and M. M. Tentzeris are with the School of ECE, Georgia Institute of Technology, Atlanta, GA 30332 USA (e-mail: stephen.kim@gatech.edu; joylaskar@gmail.com).

C.-H. Lee is with Qualcomm, San Diego CA 92122 USA.

J. Choi and F. Bien are with the Ulsan National Institute of Science and Technology (UNIST), Ulsan, South Korea.

S. Beck is with Texas Instruments, Dallas, TX 75243 USA.

Color versions of one or more of the figures in this paper are available online at http://ieeexplore.ieee.org.

Digital Object Identifier 10.1109/LMWC.2011.2170828
}

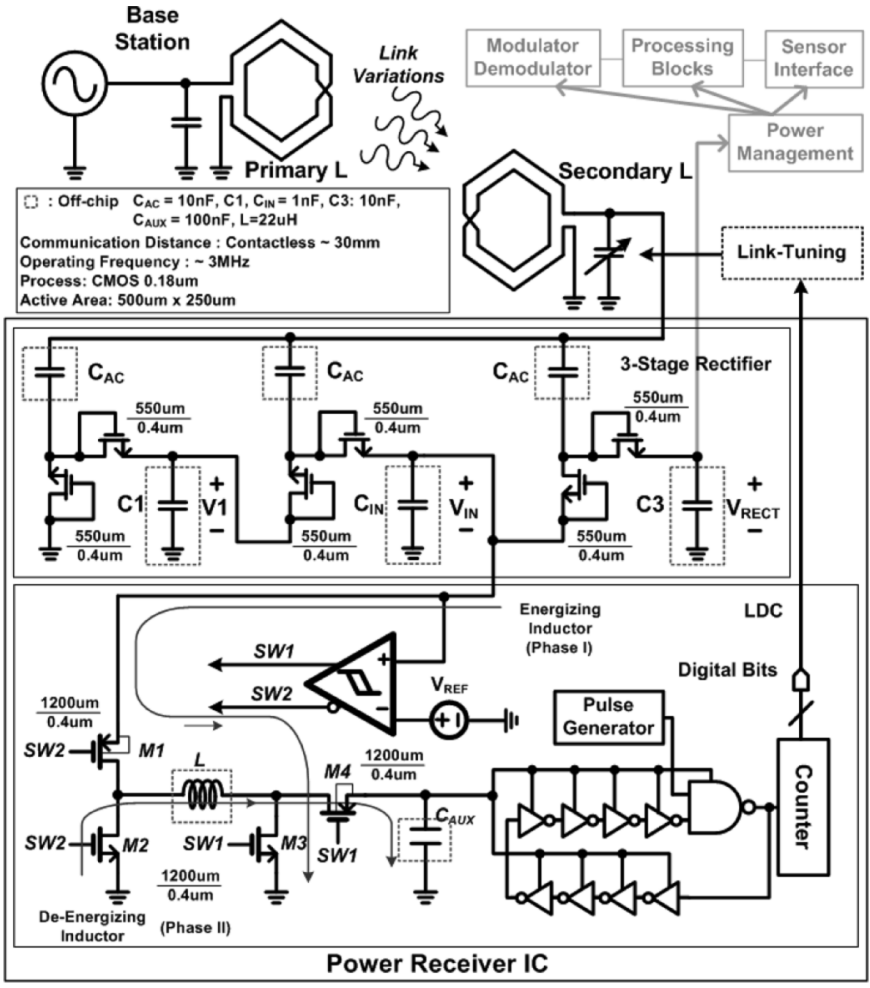

Fig. 1. Block diagram of a tunable power receiver prototype with the proposed LDC in an inductively coupled wireless sensor system.

evaluated and compensated, a wireless sensor device would consume unnecessary energy for a given task or too little energy to meet the requirement.

In [3]-[5], adaptive link-compensating schemes have been proposed. However, in [3], [4], results feature a fixed resolution determined by the physical size of the capacitor bank, and data/ power transmission must be interrupted during compensation, and [5] requires external assessment block for link evaluation.

In this letter, a novel non-interruptive link-variation-to-digital converter (LDC) is proposed for wireless sensor applications. The proposed LDC translates link-variations into digital bits that can be easily exploited in compensating the link variations. A power receiver IC with a proposed circuit is designed and fabricated in $0.18 \mu \mathrm{m}$ CMOS technology to verify the LDC operation.

\section{Proposed Link-VARIATION-TO-Digital-ConVERTER}

Fig. 1 shows the block diagram of the proposed LDC attached to a conventional wireless sensor device, which often includes a modulator and a demodulator for communication, a 

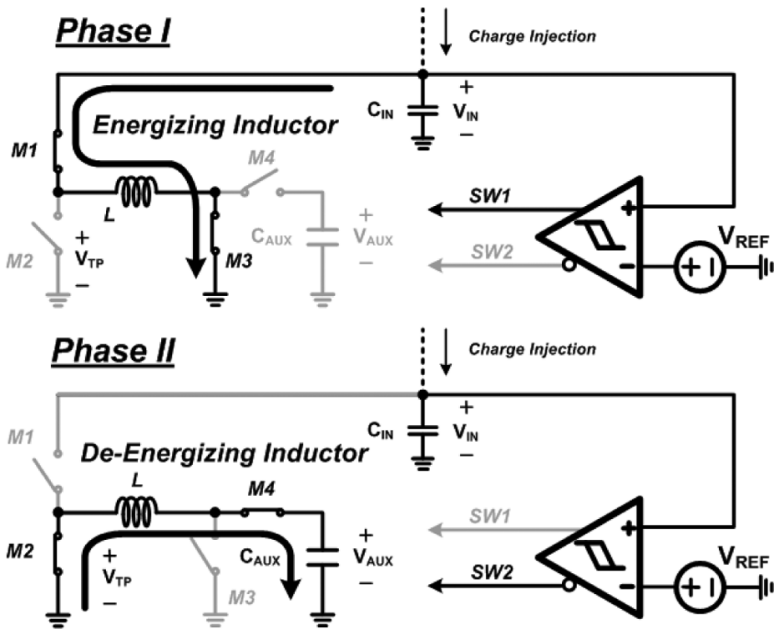

Fig. 2. Operating mechanism of the LDC power chain.

processing unit, a sensor unit, and a power management unit shown in gray solid lines. The link evaluation should have minimal disturbance on the original system functionality, and the outputs are preferred in digital. To achieve these goals, a non-inverting buck-boost power chain and a multi-resolution counting analog-to-digital converter (ADC) have been adopted in the proposed LDC.

The buck-boost chain consists of switches (M1, M2, M3, and M4), an inductor (L), and a hysteresis comparator. When $C_{\mathrm{IN}}$ is charged beyond the hysteresis comparator turn-on threshold voltage, Phase I begins as shown in Fig. 2. During Phase I, the comparator closes $\mathrm{M} 1$ and $\mathrm{M} 3$, and some of the charges in $C_{\mathrm{IN}}$ are discharged through L. As $\mathrm{L}$ gets energized, $C_{\mathrm{IN}}$ loses its charges, and $V_{\mathrm{IN}}$ decreases. When $V_{\mathrm{IN}}$ is below the turn-off threshold voltage, the comparator opens up M1 and M3 and closes M2 and M4 (the beginning of Phase II). During Phase II, the previously energized $\mathrm{L}$ dumps its remaining magnetic charge to $C_{\mathrm{AUX}}$. When $V_{\mathrm{IN}}$ charges up beyond the switching threshold, Phase II ends, and Phase I repeats.

As link variations influence the charge flow rate in $C_{\mathrm{IN}}$, the buck-boost chain translates the charge flow rate into a ratio of a Phase I duration, $t_{\mathrm{on}}$, to a Phase II duration, $t_{\text {off }}$; since the charge injection originates from the voltage multiplier, the sum of the two duration stays constant. From the principle of inductor voltsecond balance [6], we can see that $V_{\mathrm{AUX}}$ is strongly correlated with link-variations

$$
\begin{aligned}
\frac{V_{I N} t_{o n}}{L} & =\frac{V_{A U X} t_{o f f}}{L} \rightarrow V_{A U X}=\frac{t_{o n}}{t_{o f f}} \\
V_{I N} & =\frac{D}{1-D} V_{I N}
\end{aligned}
$$

where $D=t_{\text {on }} /\left(t_{\text {on }}+t_{\text {off }}\right)$ and $V_{\text {IN }}$ is proportional to the charge flow rate.

The digitizer in the LDC is realized with a ring oscillator and a digital counter. The supply of the oscillator, which consists of eight inverters and a NAND for an enable function, is connected to $V_{\mathrm{AUX}}$. The oscillation frequency is proportional to $V_{\mathrm{AUX}}$, and the counter converts the frequency into a digital code that represents the strength of $V_{\mathrm{AUX}}$. The counting duration is controlled

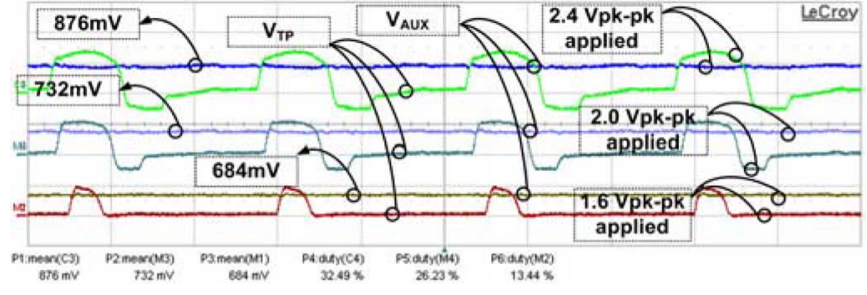

Fig. 3. Duty cycles and power chain output voltages for $2 \mathrm{MHz}$ input AC amplitudes of 2.4, 2.0, and 1.6 Vpk-pk applied to the power receiver.

by the width of a pulse from the pulse generator. This duration determines how much energy should be invested for the link-evaluation; a longer period of counting can result in a finer resolution.

\section{TunABle Power Receiver PRototype}

The tunable power receiver prototype consists of an off-chip tunable L-C network, a three-stage rectifier, and a proposed LDC block. The L-C network is realized with a flexible printed circuit board (FPCB) inductor and film-trim capacitors. The FPCB inductor was designed to have Q-factor above 100 and inductance of $15 \mu \mathrm{H}$. The physical dimension of the FPCB inductor is about $45 \mathrm{~mm} \times 45 \mathrm{~mm}$. For the prototype verification, the same FPCB inductors are used for the primary and the secondary coils. The three-stage rectifier is implemented with diode-connected transistors and forms the main energy path.

The amount of dc energy harvested at $\mathrm{C} 3$ will be affected by the link-variations in the inductively coupled link; mismatches in the resonant matching networks and inductor misalignments would be major cause for the link-variations. Therefore, with the power receiver prototype, realistic link-variations can be emulated and the functionality of the proposed LDC can be tested. Since the buck-boost power chain in the LDC isolates the remaining evaluation blocks from the main energy path, the input energy can be continuously harvested even during the link-evaluation. After the evaluation, the LDC is disabled, and the evaluation results are applied to the link-tuning operation in which a simple binary algorithm adjusts the off-chip tunable capacitor banks.

\section{MEASUREMENTS}

The combination of the FPCB inductors and the capacitor banks has a resonant frequency range of 1-5 MHz. Fig. 3 shows the power chain output, $V_{\mathrm{AUX}}$, and the corresponding duty cycle waveforms, $V_{\mathrm{TP}}$, for three input amplitudes, $2.4 \mathrm{~V}_{\mathrm{pk}-\mathrm{pk}}$, 2.0 $\mathrm{V}_{\mathrm{pk}-\mathrm{pk}}$, and $1.6 \mathrm{~V}_{\mathrm{pk}-\mathrm{pk}}$, applied to the prototype. As $2 \mathrm{MHz}$ signals are applied to the receiver with pulse periods of $2.5 \mu \mathrm{s}$, $5 \mu \mathrm{s}$, and $10 \mu \mathrm{s}$, the rectifier output, $V_{\mathrm{RECT}}$, and the LDC outputs are plotted in Fig. 4. The higher counting period is, the finer input amplitude can be differentiated.

To verify the link-evaluation capability under component mismatches, a $2 \mathrm{MHz}$ signal is transmitted through a fixed primary resonant network to a $2 \mathrm{~mm}$-away secondary resonant network, and the rectifier voltages and the LDC outputs are measured with various tuning capacitor values in Fig. 5. To emulate realistic inductor misalignment situations, the proposed 


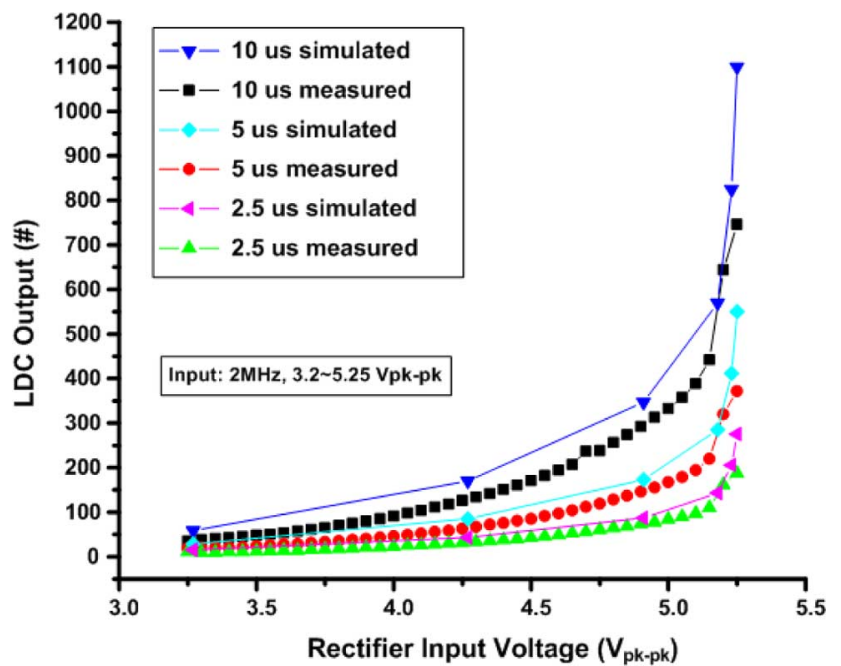

Fig. 4. LDC outputs when $2 \mathrm{MHz}$ sinusoidal signals with $3.2-5.25 \mathrm{~V}_{\mathrm{pk}-\mathrm{pk}}$ are applied to the input of the rectifier.

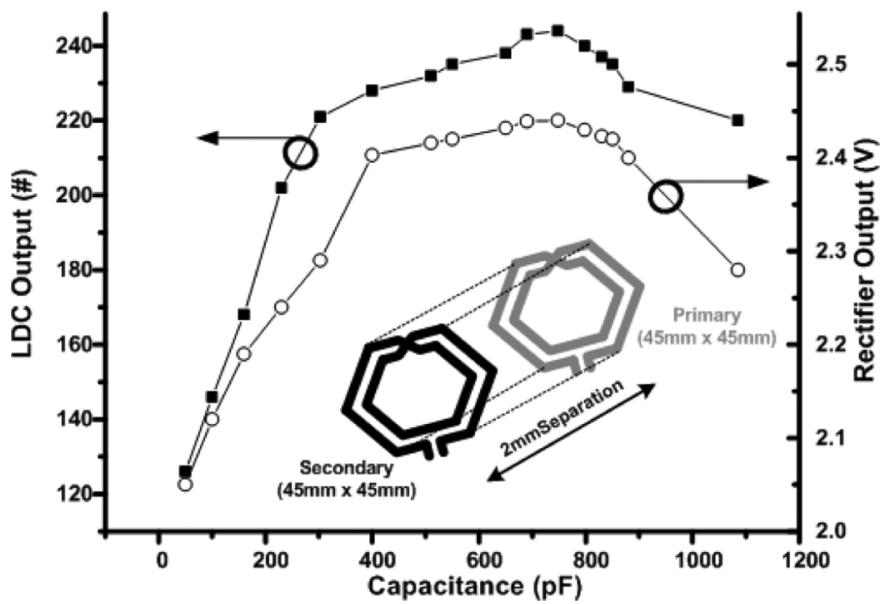

Fig. 5. Tuning capacitance value versus LDC digital outputs and corresponding rectifier output voltages.

power receiver was tested with five different inductor alignments. While the coordinate of the primary inductor is fixed, the secondary inductor is offset by $2 \mathrm{~mm}$ distance in either/both $X$-direction and $Y$-direction. For the inductor misalignments, the LDC outputs are measured with various capacitor values, as shown in Fig. 6.

The active area of the prototype, implemented in CMOS $0.18 \mu \mathrm{m}$, is $500 \mu \mathrm{m}$-by- $250 \mu \mathrm{m}$. Fig. 7 shows the die photo of the prototype and an inductor misalignment that has been tested.

\section{CONCLUSION}

In practical wireless sensor applications, unpredictable variations in the wireless link inevitably degrade the overall system performance. Evaluating the link variations in real-time is highly desirable for more reliable and efficient operations. This work presents a unique non-interruptive link-variation sensing technique that can provide in situ multi-resolution assessments.

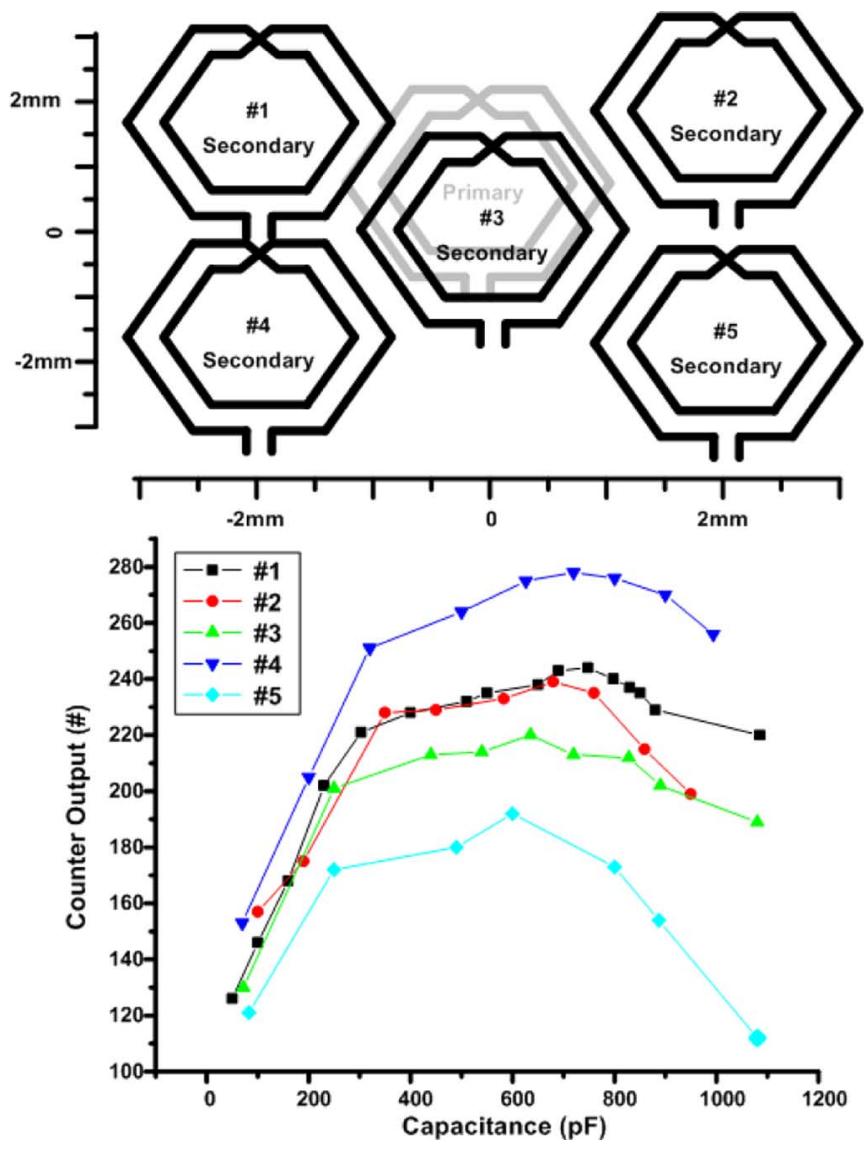

Fig. 6. Tuning capacitance value versus LDC digital outputs under various inductor misalignments.
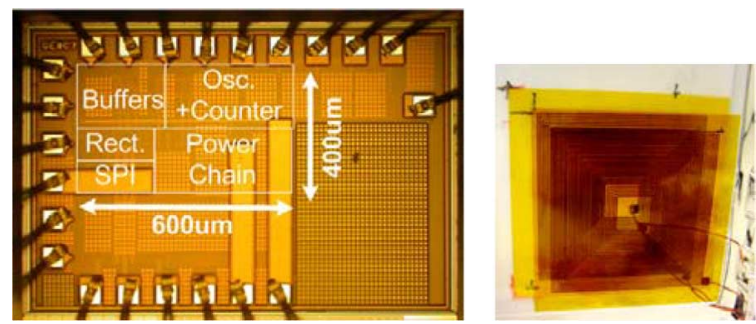

Fig. 7. Die micrograph of the power receiver prototype and an example of an inductor misalignment.

\section{REFERENCES}

[1] I. F. Akyildiz, W. Su, and Y. Sankarasubramaniam, "Wireless sensor networks: A survey," Comp. Netw., vol. 38, pp. 393-422, 2002.

[2] B. W. Cook, S. Lanzisera, and K. S. J. Pister, "SoC issues for RF smart dust," Proc. IEEE, vol. 94, no. 6, pp. 1177-1196, Jun. 2006.

[3] J. Yoo, S. Lee, and H.-J. Yoo, "A $1.12 \mathrm{pJ} / \mathrm{b}$ inductive transceiver with a fault-tolerant network switch from multi-layer wearable body area network applications," IEEE J. Solid-State Circuits, vol. 44, no. 11, pp. 2999-3010, Nov. 2009.

[4] S. Lee, J. Yoo, H. Kim, and H.-J. Yoo, "A dynamic real-time capacitor compensated inductive coupling transceiver for wearable body sensor network," in Symp. VLSI Circuits Dig. Tech. Papers, Jun. 2009, pp. $42-43$.

[5] S. O'Driscoll, A. Poon, and T. H. Meng, "A mm-sized implantable power receiver with adaptive link compensation," in IEEE ISSCC Dig. Tech. Papers, Feb. 2009, pp. 294-295.

[6] R. W. Erickson and D. Maksimovic, Fundamentals of Power Electronics, 2nd ed. Norwell, MA: Kluwer, 2001, pp. 13-35. 